\title{
On underground music and scientometrics
}

\author{
Kolos Molnár ${ }^{1,2 *}$ \\ ${ }^{1}$ Department of Polymer Engineering, Faculty of Mechanical Engineering, Budapest University of Technology and \\ Economics, Müegyetem rkp. 3-9, H-1111 Budapest, Hungary \\ ${ }^{2}$ MTA-BME Research Group for Composite Science and Technology, Múegyetem rkp. 3, H-1111 Budapest, Hungary
}

What made me wonder is that doing underground music and doing science surprisingly have many common features. A small group of skilled people produces projects together in a certain genre of their preference. The audience mainly consists of other musicians, and a few fans. The band members pay for their instruments, doing that from their family budget.

When it comes to a gig, the venue generates income from selling tickets and drinks, but do not give the musicians a single penny (subscription model). Besides that, the 'pay to play' model also exists, in which the band takes the risk of selling tickets (see APC model). Either way, these musicians never get rich from their 'publishing' activity.

Now, instead of sharing the revenues, try to convince the musicians that this whole system helps them become more popular. We can quantify their impact, and assign numbers to them as a kind of recognition. Let us track how many people streamed the band's songs (number of reads) and how many other musicians mentioned their works (number of citations). The clubs can also get ratings based on their impact (let's say c-factor). And, for instance, let's say the 'b-index' means there are a number of $B$ other bands, each mentioned at least $\mathrm{B}$ number of songs of the actual band as their influence. We can begin to construct new musicometric indices. But, this system suffers from serious issues; just to mention three:

1. I stated that Voivod, a Canadian heavy metal band influenced my $(b$-index $=4)$ band. David Grohl from the Grammy-award-winning band Foo Fighters did the same. Does my humble opinion have the same value?

2. My band's b-index reached 4, only because $70 \%$ of the citing works criticized me as being a clumsy

\footnotetext{
${ }^{*}$ Corresponding author, e-mail: molnar@pt.bme.hu
} (c) BME-PT guitarist (which truly I am). Besides, 20\% admired the drummer, not me. The other $10 \%$ were our friends. I did not ask them to do so, but they tried to make me more popular this way.

3. At my previous show, the $\mathrm{Q} 1$ venue had a high cfactor in 2020, but only 3 people came to see my show (one of which was independent). Besides, the club's profile has changed since last year.

Now, let us put some pressure on our musicians. If they do not reach a certain c-factor and b-index they will not get support for their rehearsal room (garage) rental from the city government. Do they still want to make good music or have we just constructed a deteriorative incentive? Similar to this, scientometrics give a lot of useful information, but relying solely on those is greatly misleading.

These amateur musicians are aware that each kid has a different background: information access issues, financial issues, language issues, etc. and they are completely OK with that. They do not strive to pump up such numbers, but practice, evolve, and support one another. And if the same, non-profit musicians operate the concert venue then they understand all these issues. Express Polymer Letters provides an entirely free platform within the polymer 'genre'. We want to enjoy quality music together, after all.

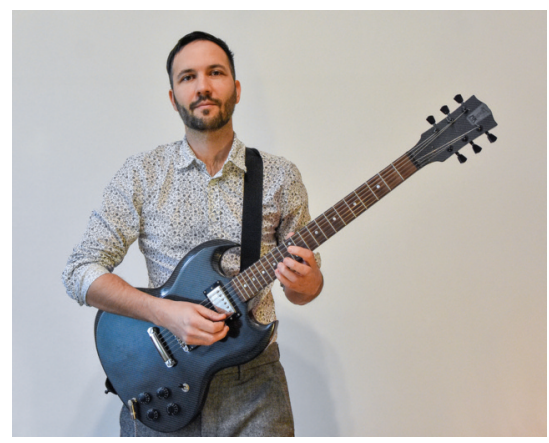

Dr. Kolos Molnár topic editor with his self-made carbon fiber reinforced composite guitar 